\title{
All-cause excess mortality and COVID-19-related deaths in Iran
}

\author{
Hooman Tadbiri ${ }^{1}$, Maziar Moradi-Lakeh*2(1), Mohsen Naghavi ${ }^{3}$ \\ Received: 19 Jun 2020 \\ Published: 15 Jul 2020
}

\section{Abstract}

Background: Iran reported its first COVID-19 deaths on February 19, 2020 and announced 1284 deaths with a laboratoryconfirmed SARS-CoV-2 infection by March 19, 2020 (end of the winter $1398 \mathrm{SH}$ ). We estimated all-cause excess mortality, compared to the historical trends, to obtain an indirect estimate of COVID-19-related deaths.

Methods: We assembled time series of the seasonal number of all-cause mortalities from March 21, 2013 (spring of 1392 SH) to March 19, 2020 (winter $1398 \mathrm{SH}$ ) for each province of Iran and nationwide with the vital statistics data from the National Organization for Civil Registration (NOCR). We estimated the expected seasonal mortality and excess mortality (the difference between the number of registered and expected deaths). Moreover, we reviewed the provincial number of confirmed cases of COVID-19 to assess their association with excess deaths.

Results: The results of our analysis showed around 7507 (95\% CI: 3,350 - 11,664) and 5180 (95\% CI: 1,023 - 9,337) all-cause excess mortality in fall and winter, respectively. There were 3778 excess deaths occurred in Qom, Gilan, Mazandaran, and Golestan provinces in the winter, all among the COVID-19 epicenters based on the number of confirmed cases.

Conclusion: We think most of the excess deaths in the winter were related to COVID-19. Also, we think the influenza epidemic might have been the main reason for the excess mortality in the fall and parts of excess deaths in the winter of $1398 \mathrm{SH}$. Moreover, a review of all available clinical and paraclinical records and through analyses of the surveillance data for severe acute respiratory infections (SARI) can help to obtain a more accurate estimate of COVID-19 mortality.

Keywords: COVID-19, SARS-CoV-2, Coronavirus, Iran, Mortality, Excess mortality, Excess deaths

\author{
Conflicts of Interest: None declared \\ Funding: None \\ *This work has been published under CC BY-NC-SA 1.0 license. \\ Copyright $\odot$ Iran University of Medical Sciences
}

Cite this article as: Tadbiri H, Moradi-Lakeh M, Naghavi M. All-cause excess mortality and COVID-19-related deaths in Iran. Med J Islam Repub Iran. 2020 (15 Jul);34:80. https://doi.org/10.47176/mjiri.34.80

\section{Introduction}

A novel coronavirus, SARS-CoV-2, was first reported in December 2019 in Wuhan, China (1). The disease caused by this virus was named COVID-19 (2). The virus quickly spread in the world, and the World Health Organization (WHO) characterized COVID-19 as a pandemic on March 11, 2020 (3). The number of reported COVID-19

Corresponding author: Dr Maziar Moradi-Lakeh, moradilakeh.m@iums.ac.ir

1. Johns Hopkins Bloomberg School of Public Health, Baltimore, MD, USA

2. Preventive Medicine and Public Health Research Center, Psychosocial Health Research Institute, Iran University of Medical Sciences, Tehran, Iran

3. Institute for Health Metrics and Evaluation, University of Washington, Seattle, WA, USA cases in each country is a function of the real number of cases, health system capacity to detect cases, and its transparency to report correct measures. The capacity of the health system for detecting cases is driven by different factors, such as availability and accessibility of diagnostic (and screening) tests and healthcare services, accuracy of

$\uparrow$ What is "already known" in this topic:

- In the winter 1398 SH (March 19, 2020), the Ministry of Health and Medical Education of Iran reported 1284 deaths in confirmed cases of COVID-19.

- Fatalities due to some other causes of deaths, such as traffic injuries, have changed during the COVID-19 epidemic.

$\rightarrow$ What this article adds:

- Compared to the historical trend, 7507 (95\% CI: 3,350$11,664)$ and $5180(95 \%$ CI: 1,023-9,337) excess deaths were estimated for the fall and winter $1398 \mathrm{SH}$, respectively.

- We believe most of the excess deaths in the winter were related to COVID-19, although the continuation of the influenza epidemic in the fall might have contributed to the excess deaths in the winter. 
laboratory tests, and raising public awareness about the disease, and establishing a process for seeking care $(4,5)$. Due to the disastrous nature of this epidemic and the risk of collapse in the health system, many of the suspected COVID-19 cases with mild to moderate symptoms and asymptomatic individuals exposed to known cases were encouraged to self-isolate themselves at home, even in the high-income countries (6). Thus, the number of confirmed cases does not necessarily reflect the real number of cases.

On February 19, 2020, Iran reported 2 confirmed cases of COVID-19 for the first time $(7,8)$ and reported its first deaths due to COVID-19 on the same day $(8,9)$. By the end of the winter $1398 \mathrm{SH}$ (March 19, 2020), the number of reported cases and deaths due to COVID-19 in Iran reached to 18407 and 1284 , respectively $(10,11)$. The Ministry of Health and Medical Education (MoHME) reports the number of deaths occurring in persons with laboratory-confirmed SARS-CoV-2 infection. However, every person who had died of COVID-19 was not suspected to have COVID-19, diagnostic testing was not available to every person who was suspected to have the disease, and many of those who got tested, had falsenegative results. A report estimated the sensitivity of COVID-19 testing in Iran to be around 51.4\% (95\% CI: $49.6 \%-53.1 \%)(12)$.

Death registration systems are facing several challenges, such as undercoverage, incompleteness, improper allocation of causes of deaths, a high percentage of garbage codes, and delays in registration. In the middle of an epidemic, the performance of a death registration system may be even worse than usual. For instance, diagnostic tests might not be available, or their results might not be ready before the disposal of deceased cases. Also, the healthcare staff might be overwhelmed with clinical care, which increases errors in the allocation of causes of deaths. However, the number of deaths is expected to be a more robust indicator of the spread of the disease compared to the number of incident cases.

An "unusual rate of death" in Iran in the fall $1398 \mathrm{SH}$ (September 23, 2019 - December 21, 2019) and the winter 1398 SH (December 22, 2019 - March 19, 2020) was also noticed by other authors $(13,14)$. The aim of this study was to provide an estimate of the all-cause excess mortality (the excess number of observed deaths compared to the expected seasonal number based on the historical trends) in Iran in fall and winter of $1398 \mathrm{SH}$. Estimating all-cause excess mortality from the national vital statistics data may provide a good assessment of the burden of the epidemics on mortality $(15,16)$.

\section{Methods}

We assembled time series of the seasonal number of allcause mortality from the spring 1392 SH (March 21, 2013) to the winter $1398 \mathrm{SH}$ (March 19, 2020) for each province and nationwide using the vital statistics data from the National Organization for Civil Registration (NOCR) (17).

First, we estimated the expected number of deaths in the country for each season using these time series. We fitted a linear regression model with a term for secular trend and terms for seasonal variations to the time series data. We detected extremely influential observations using the DFITS statistic (18) and fitted the model again to the data without those observations. Removing those artifacts was a necessary step to obtain a more accurate estimate of the baseline expected mortality (19).

Then, we estimated the excess deaths in the country and in each province for the seasons of $1398 \mathrm{SH}$ (March 20, 2019 - March 19, 2020) with the observed number of deaths in the previous years. We used the model above and data from prior seasons to estimate the number of expected deaths in each season. Next, we calculated the difference between the estimate from our model and the number of deaths registered by NOCR in that season. We used the standard errors of the forecast to calculate confidence intervals. Similar approaches have been widely used to calculate excess all-cause mortality during pandemics $(15,20)$.

Excess mortality is considered statistically significant when $95 \%$ confidence interval does not include zero. In this study all analyses were performed using Stata version $15.1(21)$.

We extracted the provincial number of persons with a laboratory-confirmed COVID-19 in the winter $1398 \mathrm{SH}$ from the daily briefings of MoHME (22). Number of COVID-19 deaths is not publicly available at the provincial level; thus, we plotted the number of excess deaths in the winter in each province against the number of confirmed COVID-19 cases in that season in the province to assess any association.

To have an idea about the potential decline in the number of deaths due to other causes, we extracted the number of deaths due to traffic injuries from the periodical reports of the Iranian Legal Medicine Organization (ILMO) (23). In addition to annual reports, ILMO has reports on the number of deaths for specific months of each year, including the last month of winter in each Solar Hijri calendar year, which is compatible with March $20^{\text {th }} / 21^{\text {st }}$ to March $19^{\text {th }} / 20^{\text {th }}$ of the Georgian calendar.

\section{Results}

In the year $1398 \mathrm{SH}$ (March 20, 2019 - March 19, 2020), 395392 deaths were registered by NOCR, which was 18368 more than the previous year, $1397 \mathrm{SH}$ (March 21, 2018 - March 19, 2019). The registered number of deaths in the spring and summer of $1398 \mathrm{SH}$ was not significantly different from the expected numbers; however, the numbers of death tolls were beyond the expected numbers both in the fall and winter of $1398 \mathrm{SH}$ (Fig. 1).

According to the NOCR, 103422 deaths were registered in the fall season of $1398 \mathrm{SH}$ in Iran, which was 9559 more than the fall $1397 \mathrm{SH}$, showing 7507 excess deaths (95\% CI: 3350 - 11 664), compared to the expected baseline for the fall $1398 \mathrm{SH}$.

In our analysis by province, we found that 19 of 31 provinces had a significantly higher number of registered deaths in the fall $1398 \mathrm{SH}$ compared to the expected baseline for that season. More details are available in Table 1.

According to the NOCR, 105787 deaths were registered in the winter $1398 \mathrm{SH}$ in Iran, which indicated 4577 more 


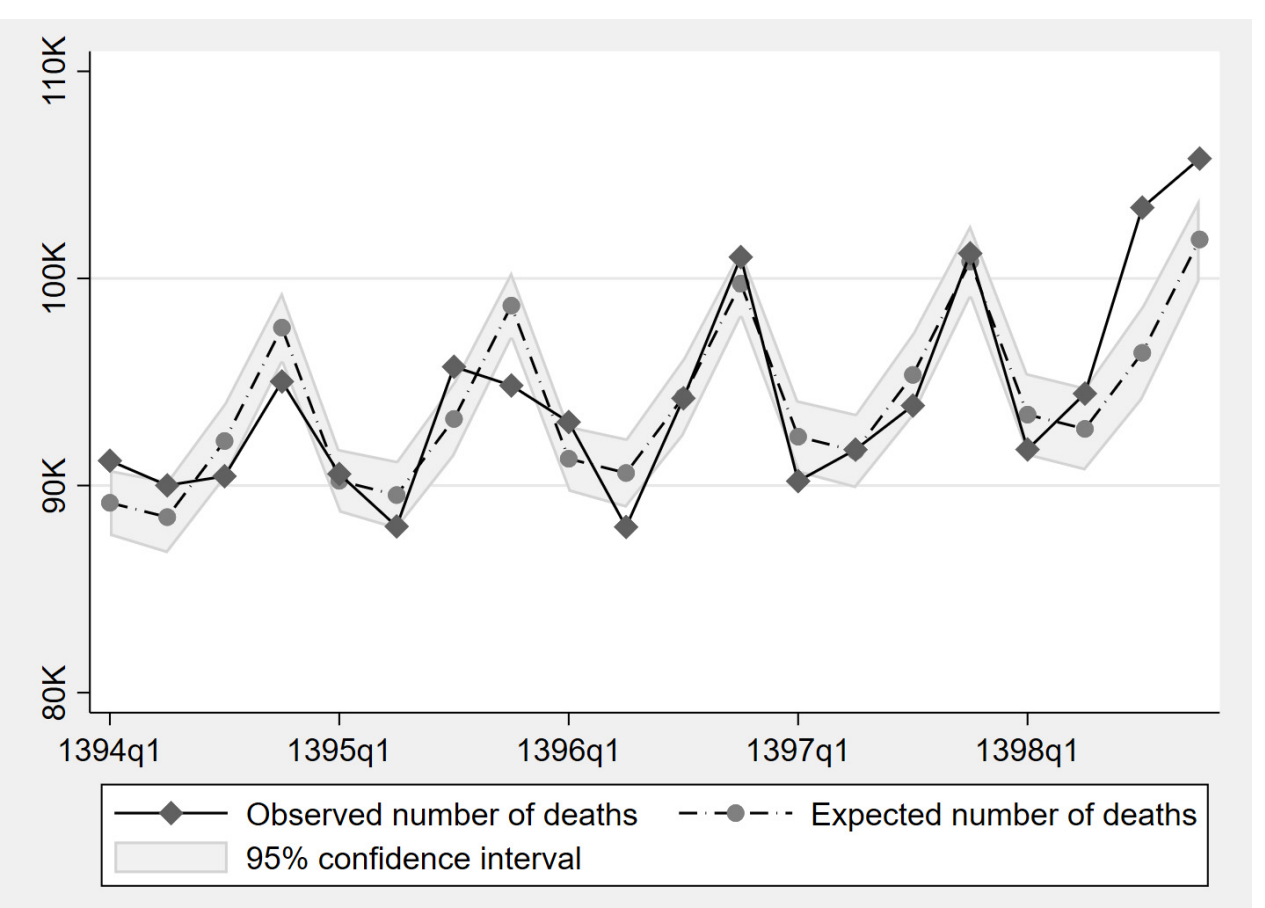

Note: q1 refers to the spring season.

Fig. 1. Observed and expected number of deaths at the national level in Iran based on the historical trend (1394-1398 SH, 2015-2020)

Table 1. Estimated excess deaths by province based on historical trends (1398 SH, 2019-20)

\begin{tabular}{|c|c|c|c|c|c|c|}
\hline \multirow[t]{3}{*}{ Province } & \multicolumn{3}{|c|}{ Fall } & \multicolumn{3}{|c|}{ Winter } \\
\hline & \multirow[t]{2}{*}{ Difference* } & \multicolumn{2}{|c|}{ 95\% Confidence Interval } & \multirow[t]{2}{*}{ Difference* } & \multicolumn{2}{|c|}{$95 \%$ Confidence Interval } \\
\hline & & Lower Bound* & Upper Bound* & & Lower Bound* & Upper Bound* \\
\hline Alborz & $426(14.19 \%)$ & $108(3.58 \%)$ & $745(24.81 \%)$ & $351(11.43 \%)$ & $55(1.79 \%)$ & $647(21.06 \%)$ \\
\hline Ardabil & $114(6.24 \%)$ & $-61(-3.34 \%)$ & $289(15.83 \%)$ & $45(2.50 \%)$ & $-133(-7.46 \%)$ & $222(12.46 \%)$ \\
\hline Azerbaijan, East & $732(11.82 \%)$ & $455(7.35 \%)$ & $1010(16.29 \%)$ & $-118(-2.04 \%)$ & $-395(-6.85 \%)$ & $159(2.77 \%)$ \\
\hline Azerbaijan, West & $333(8.16 \%)$ & $118(2.91 \%)$ & $547(13.42 \%)$ & $24(0.60 \%)$ & $-190(-4.72 \%)$ & $238(5.91 \%)$ \\
\hline Bushehr & $162(13.04 \%)$ & $55(4.44 \%)$ & $269(21.64 \%)$ & $-21(-1.80 \%)$ & $-128(-10.84 \%)$ & $85(7.24 \%)$ \\
\hline Chaharmahal and Bakhtiari & $19(1.86 \%)$ & $-89(-8.69 \%)$ & $128(12.41 \%)$ & $45(4.26 \%)$ & $-58(-5.51 \%)$ & $149(14.03 \%)$ \\
\hline Fars & $346(5.97 \%)$ & $82(1.42 \%)$ & $609(10.52 \%)$ & $-8(-0.13 \%)$ & $-277(-4.79 \%)$ & $262(4.53 \%)$ \\
\hline Gilan & $265(5.98 \%)$ & $54(1.23 \%)$ & $475(10.73 \%)$ & $1524(25.68 \%)$ & $1314(22.14 \%)$ & $1735(29.22 \%)$ \\
\hline Golestan & $258(10.14 \%)$ & $168(6.58 \%)$ & $349(13.71 \%)$ & $535(18.17 \%)$ & $445(15.09 \%)$ & $626(21.25 \%)$ \\
\hline Hamedan & $244(8.57 \%)$ & $-24(-0.85 \%)$ & $512(17.99 \%)$ & $-22(-0.82 \%)$ & $-302(-11.14 \%)$ & $258(9.50 \%)$ \\
\hline Hormozgan & $142(8.02 \%)$ & $-22(-1.26 \%)$ & $306(17.30 \%)$ & $-38(-2.09 \%)$ & $-207(-11.53 \%)$ & $132(7.36 \%)$ \\
\hline Ilam & $62(9.09 \%)$ & $-9(-1.26 \%)$ & $134(19.45 \%)$ & $-54(-8.48 \%)$ & $-128(-20.23 \%)$ & $21(3.27 \%)$ \\
\hline Isfahan & $746(11.35 \%)$ & $255(3.87 \%)$ & $1238(18.83 \%)$ & $487(7.24 \%)$ & $-5(-0.07 \%)$ & $979(14.54 \%)$ \\
\hline Kerman & $72(1.98 \%)$ & $-253(-6.98 \%)$ & $396(10.94 \%)$ & $-111(-3.02 \%)$ & $-429(-11.65 \%)$ & $206(5.60 \%)$ \\
\hline Kermanshah & $346(11.28 \%)$ & $84(2.75 \%)$ & $607(19.81 \%)$ & $78(2.70 \%)$ & $-228(-7.88 \%)$ & $385(13.28 \%)$ \\
\hline Khorasan, North & $268(19.86 \%)$ & $154(11.41 \%)$ & $382(28.31 \%)$ & $92(7.43 \%)$ & $-22(-1.80 \%)$ & $206(16.66 \%)$ \\
\hline Khorasan, Razavi & $755(8.82 \%)$ & $326(3.81 \%)$ & $1183(13.82 \%)$ & $-421(-5.38 \%)$ & $-839(-10.72 \%)$ & $-2(-0.03 \%)$ \\
\hline Khorasan, South & $155(13.79 \%)$ & $19(1.71 \%)$ & $291(25.87 \%)$ & $1(0.14 \%)$ & $-134(-13.73 \%)$ & $137(14.01 \%)$ \\
\hline Khuzestan & $537(9.26 \%)$ & $137(2.36 \%)$ & $938(16.16 \%)$ & $-100(-1.79 \%)$ & $-490(-8.81 \%)$ & $291(5.23 \%)$ \\
\hline Kohgiluyeh and Boyer-Ahmad & $19(2.58 \%)$ & $-73(-9.92 \%)$ & $111(15.08 \%)$ & $-36(-5.09 \%)$ & $-126(-17.70 \%)$ & $53(7.52 \%)$ \\
\hline Kurdistan & $206(10.45 \%)$ & $34(1.71 \%)$ & $379(19.19 \%)$ & $20(1.07 \%)$ & $-152(-7.95 \%)$ & $193(10.10 \%)$ \\
\hline Lorestan & $131(5.58 \%)$ & $-94(-4.00 \%)$ & $355(15.16 \%)$ & $24(1.05 \%)$ & $-200(-8.71 \%)$ & $248(10.81 \%)$ \\
\hline Markazi & $105(5.12 \%)$ & $-42(-2.04 \%)$ & $251(12.28 \%)$ & $166(7.86 \%)$ & $17(0.79 \%)$ & $315(14.93 \%)$ \\
\hline Mazandaran & $337(7.40 \%)$ & $110(2.41 \%)$ & $564(12.40 \%)$ & $1002(18.99 \%)$ & $774(14.68 \%)$ & $1229(23.30 \%)$ \\
\hline Qazvin & $171(10.07 \%)$ & $35(2.07 \%)$ & $307(18.08 \%)$ & $146(8.47 \%)$ & $10(0.60 \%)$ & $283(16.35 \%)$ \\
\hline Qom & $105(6.47 \%)$ & $37(2.27 \%)$ & $173(10.67 \%)$ & $717(31.09 \%)$ & $648(28.13 \%)$ & $785(34.05 \%)$ \\
\hline Semnan & $25(2.78 \%)$ & $-61(-6.66 \%)$ & $111(12.22 \%)$ & $52(5.43 \%)$ & $-24(-2.58 \%)$ & $128(13.44 \%)$ \\
\hline Sistan and Baluchestan & $-303(-9.10 \%)$ & $-1183(-35.47 \%)$ & $576(17.28 \%)$ & $-398(-11.73 \%)$ & $-1261(-37.21 \%)$ & $466(13.75 \%)$ \\
\hline Tehran & $919(5.81 \%)$ & $105(0.67 \%)$ & $1732(10.95 \%)$ & $1011(6.03 \%)$ & $134(0.80 \%)$ & $1889(11.26 \%)$ \\
\hline Yazd & $125(9.31 \%)$ & $-2(-0.14 \%)$ & $252(18.76 \%)$ & $104(7.79 \%)$ & $-26(-1.93 \%)$ & $233(17.51 \%)$ \\
\hline Zanjan & $193(12.84 \%)$ & $53(3.57 \%)$ & $332(22.11 \%)$ & $50(3.55 \%)$ & $-90(-6.41 \%)$ & $189(13.52 \%)$ \\
\hline Iran & $7507(7.26 \%)$ & $3350(3.24 \%)$ & $11664(11.28 \%)$ & $5180(4.90 \%)$ & $1023(0.97 \%)$ & $9337(8.83 \%)$ \\
\hline
\end{tabular}

deaths than the winter $1397 \mathrm{SH}$ and 5180 excess deaths (95\% CI: 1023 - 9337) compared to the expected baseline for the winter $1398 \mathrm{SH}$.
In our analysis by province, we found that 8 provinces had a significantly higher number of registered deaths in the winter $1398 \mathrm{SH}$ compared to their expected baseline 
for that season, and 1 province had a significantly lower number of registered deaths in the winter $1398 \mathrm{SH}$ compared to its expected baseline for that season. Four leading provinces according to the percentage of excess deaths to total deaths were Qom, Gilan, Mazandaran, and Golestan (Fig. 2). The top 4 provinces according to the number of excess deaths were Gilan, Tehran, Mazandaran, and Qom.
The complete results are presented in Table 1.

The MoHME reported 1284 COVID-19 deaths in Iran until March 19, 2020 (10). The number of deaths due to COVID-19 by province was not officially reported. Figure 3 shows the association of estimated excess deaths and the number of COVID-19 confirmed cases.

The number of fatalities of traffic injuries in the last
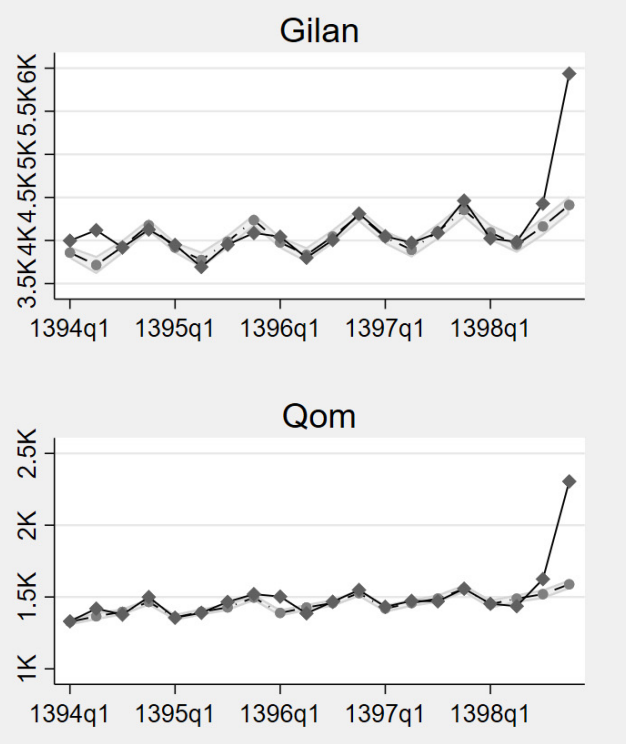

$\longrightarrow$ Observed number of deaths $95 \%$ confidence interval

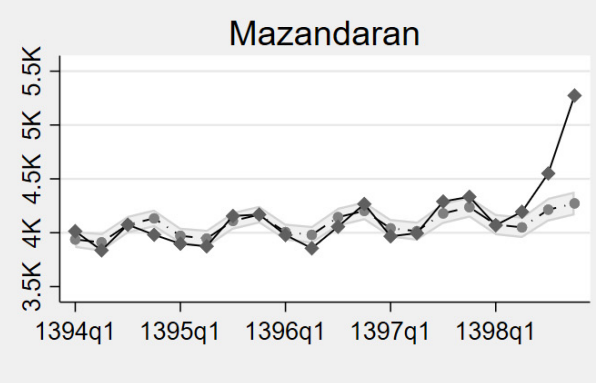

Golestan

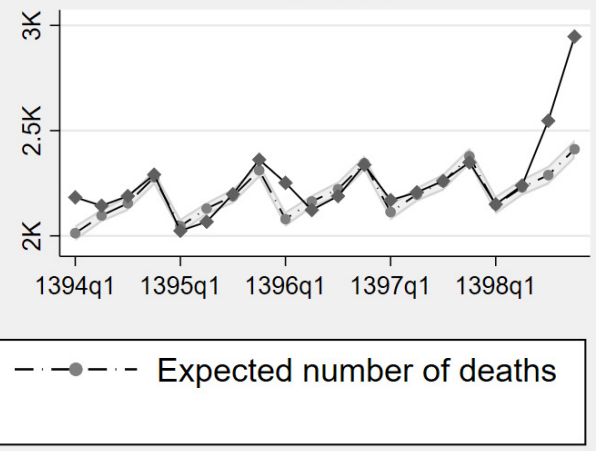

Note: q1 refers to the spring season

Fig. 2. Observed and expected number of deaths in four provinces based on their historical trends (1394-1398 SH, 2015-2020)

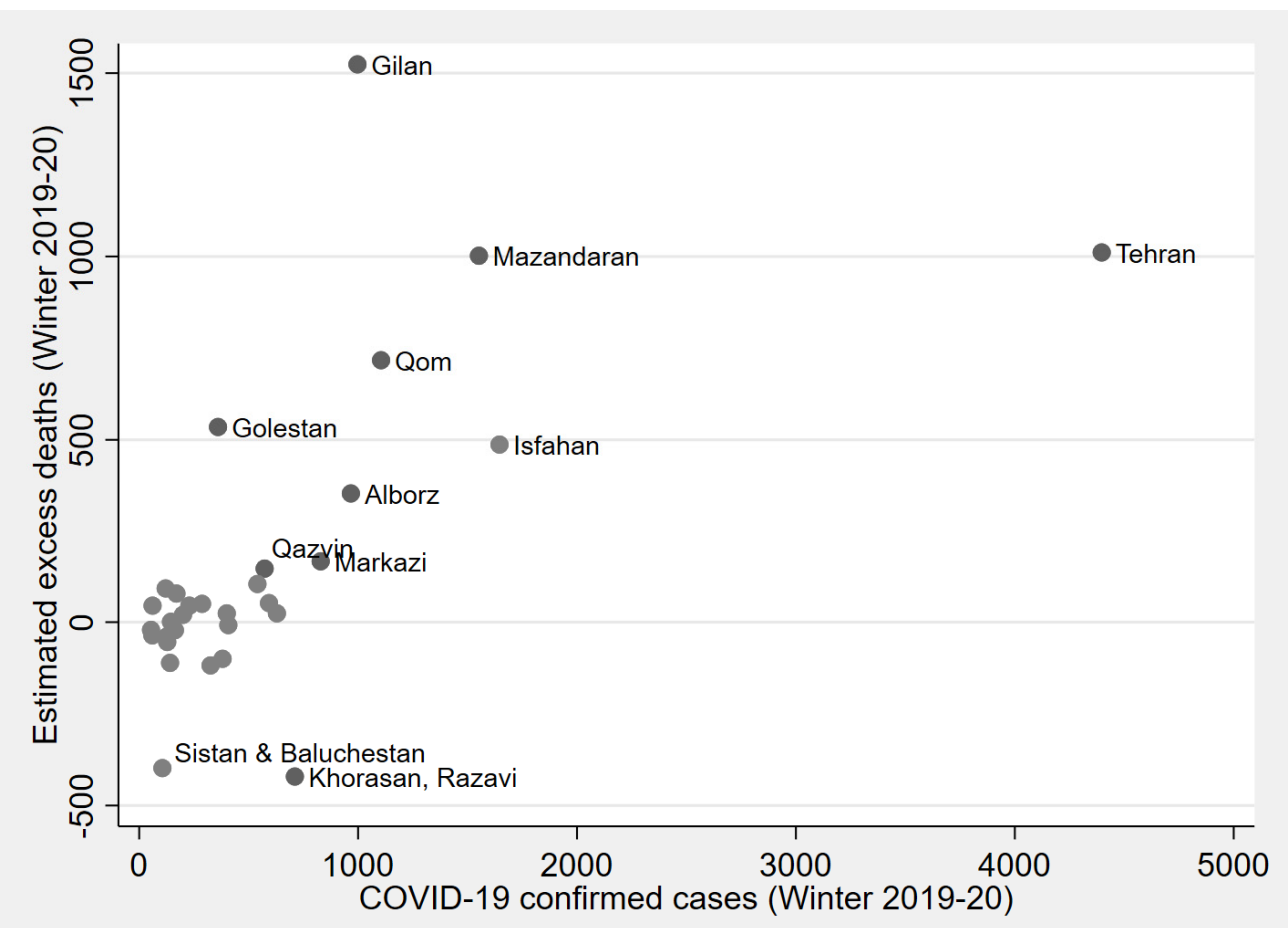

Fig. 3. Association of estimated excess deaths in the winter of 1398 SH (December 21, 2019 - March 19, 2020) and reported number of COVID-19 confirmed cases among provinces of Iran 
Table 2. Deaths due to traffic injuries in the last month of the winter compared to the total number of registered deaths in the winter (1394-1398 SH, 2015-2020)

\begin{tabular}{|c|c|c|c|}
\hline \multirow[t]{2}{*}{ Solar Hijri* (Georgian) Year } & \multirow[t]{2}{*}{ Total Deaths in the Winter } & \multicolumn{2}{|c|}{ Traffic Injury Deaths in the Last Month of the Winter } \\
\hline & & Number & Percent \\
\hline $1394(2015-16)$ & 95,026 & 1,280 & $1.35 \%$ \\
\hline $1395(2016-17)$ & 94,831 & 1,144 & $1.21 \%$ \\
\hline $1396(2017-18)$ & 101,030 & 1,179 & $1.17 \%$ \\
\hline $1397(2018-19)$ & 101,210 & 1,257 & $1.24 \%$ \\
\hline $1398(2019-20)$ & 105,787 & 1,048 & $0.99 \%$ \\
\hline
\end{tabular}

month of the winter and its percentage to the total number of deaths was lower in the winter $1398 \mathrm{SH}$ compared to previous years in Iran (Table 2).

\section{Discussion}

We applied a regression model on the seasonal time series of all-cause deaths to estimate the excess mortality in the fall and winter of $1398 \mathrm{SH}$ in Iran. The results of our analysis showed around 7507 and 5180 excess deaths in the fall and winter of $1398 \mathrm{SH}$ in Iran, respectively. A total of 3778 excess deaths occurred in the winter only in the 4 provinces of Qom, Gilan, Mazandaran, and Golestan, with the highest rise in their mortality trajectories and all among the epicenters of COVID-19 in the winter. This number is remarkably higher than the 1284 confirmed COVID-19 deaths that were officially reported for the whole country in the winter.

The most common models use weekly or monthly data to estimate excess mortality, but such data were not available for this study. We used seasonal mortality data from NOCR, which reflects the number of deaths registered in each season. This means some deaths registered in every season may have occurred before that season. The United Nations Statistical Division (USDN) estimated the coverage of death registration in Iran to be around 92\% (24). This indicator may vary among different subnational locations and periods. Due to partial closure of nonessential services, we expect to have more non-registered deaths or postponed registrations in the last month of the winter. Furthermore, we did not have access to age-specific mortality data for a more detailed and accurate estimation.

Some authors have earlier estimated the excess mortality in the fall and winter of 1398 SH in Iran by comparing the number of deaths with the 5-year average (13). That approach is more prone to overestimation due to neglecting the slight annual increase in the number of deaths, which is mainly due to population growth. We used a different approach for estimating excess deaths to address secular trends.

Considering the COVID-19 pandemic in the winter 2019-20, it is reasonable to assume that a major part of the excess deaths is due to COVID-19 or has been affected by the epidemic, and the association between excess deaths and the number of confirmed cases supports this argument. However, there are other potential causes for the difference between the expected and registered deaths. One of the potential causes is influenza; influenza is probably the main reason for excess deaths in the fall $1398 \mathrm{SH}$. The WHO had reported influenza activity "started to in- crease" in Iran based on data from October 14, 2019 to October 27, 2019 (25), "continued to increase" based on data from October 28, 2019 to November 24, 2019 (26, 27), and "remained elevated" based on data from November 25, 2019 to December 22, 2019 (28, 29). Also, there were protests in November 2019 in some of the provinces of Iran (as a reaction to the rise of fuel price) that resulted in fatalities. Different numbers have been announced as the number of the death toll from around 200 to 230 (by a government official and a member of the parliament (30, 31)), to more than 304 (by the Amnesty International (32)) and 1500 (by a news agency (33)). Even with the highest current estimate of unrest fatalities, most of the fall 1398 $\mathrm{SH}$ excess deaths remain unexplained and are most likely to be due to the influenza epidemic. Assuming that most of the extra deaths in fall are due to the outbreak of influenza, then it is reasonable to assume that influenza has partially contributed to excess deaths in the winter $1398 \mathrm{SH}$ as well. On the other hand, the number of fatalities due to traffic injuries was lower than expected, which might have been due to decreased transportation because of the COVID-19 interventions, or due to other factors not related to COVID-19 (such as the increased price of fuel or decreased general purchasing power). This factor, in addition to the potentially more than usual delayed registration of deaths at NOCR because of the social distancing interventions and partial closure of governmental organizations, may have contributed to an underestimation of excess deaths that are related to COVID-19. Experience of China (in Wuhan) shows that COVID-19 confirmed deaths might increase considerably after a retrograde comprehensive review of all clinical and paraclinical findings from different sources, such as hospitals and nursing homes (34).

Using the excess mortality has some limitations as a measure of the severity of epidemics; for instance, the number of NOCR registered deaths might change due to factors such as a change in regulations, which are completely irrelevant to health factors and the real number of deaths. There might be other reasons, not-related to COVID-19 or other factors mentioned in this report, which have contributed to excess mortality in the fall and winter of $1398 \mathrm{SH}$.

We did not have access to the provincial distribution of the number of confirmed COVID-19 deaths and surveillance data for severe acute respiratory infections (SARI); these are 2 important sources of data to assess excess deaths in the fall and winter of $1398 \mathrm{SH}$. Also, we did not have access to the age distribution of deaths in 
each province; considering age and sex distributions might increase the accuracy of models for estimating expected deaths.

\section{Conclusion}

In the winter $1398 \mathrm{SH}$, Iran experienced around 5180 excess deaths compared to the expectations based on its historical trend. Although the extension of the influenza outbreak, which started in fall, might have contributed to the excess deaths of the winter, we think most of the excess mortality of the winter was related to COVID-19. Considering a decline in some other causes of deaths, such as traffic injuries, and an expected delay in vital registration due to epidemic situation, the real number of COVID19-related deaths might be even higher. A second look and review of all clinical and paraclinical records and analysis of SARI findings can help to have a more accurate measure for the number of COVID-19-related deaths.

\section{Conflict of Interests}

The authors declare that they have no competing interests.

\section{References}

1. World Health Organization (WHO). Novel Coronavirus (2019nCoV): situation report, 1. 2020.

2. World Health Organization (WHO). Novel Coronavirus (2019nCoV): situation report, 22. 2020.

3. World Health Organization (WHO). Coronavirus disease 2019 (COVID-19): situation report, 51. 2020.

4. Goldstein ND, Burstyn I. On the importance of early testing even when imperfect in a pandemic such as COVID-19. 2020. doi:10.31219/osf.io/9pz4d.

5. Kandel N, Chungong S, Omaar A, Xing J. Health security capacities in the context of COVID-19 outbreak: an analysis of International Health Regulations annual report data from 182 countries. Lancet. 2020. doi:10.1016/S0140-6736(20)30553-5.

6. Moghadas SM, Shoukat A, Fitzpatrick MC, Wells CR, Sah P, Pandey A, et al. Projecting hospital utilization during the COVID-19 outbreaks in the United States. Proceedings of the National Academy of Sciences. 2020;117(16):9122-9126.

7. Ministry of Health and Medical Education (MoHME). Preliminary test results of two suspected coronavirus cases have been reported positive. Tehran, Iran: 2020 February 19. [cited 2020 May 22].

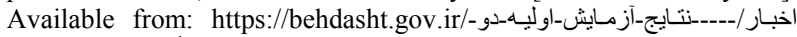

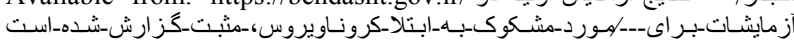
قطعيت-تشخيص-در -حال-انجام-است

8. World Health Organization (WHO). Coronavirus disease 2019 (COVID-19): situation report, 31. 2020.

9. Ministry of Health and Medical Education (MoHME). Death of two patients with novel coronavirus in Qom. Tehran, Iran: 2020 February 19. [cited 2020 May 22]. Available from:

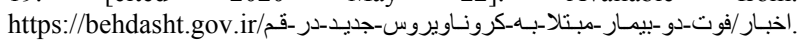
Persian.

10. Ministry of Health and Medical Education (MoHME). Identification of 1046 new patients with COVID-19 in the country. Tehran, Iran: 2020 March 19. [cited 2020 May 23]. Available from:

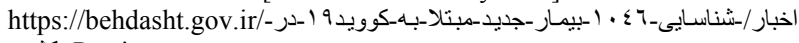
كثور. Persian.

11. World Health Organization (WHO). Coronavirus disease 2019 (COVID-19): situation report, 60. 2020.

12. COVID-19 Epidemiology Committee. Epidemiological report of COVID-19 patients in hospitals covered by Tehran University of Medical Sciences. Tehran University of Medical Sciences; 2020. [cited 2020 May 14]. Available from: http://nihr.tums.ac.ir/Item/782. Persian.

13. Ghafari M, Madani K. In search of the murder: Making sense of Iran's reported deaths. Medium; 2020 May 25. [cited 2020 May 28].
Available from: https://medium.com/@kavehmadani/in-search-of-themurder-making-sense-of-irans-reported-deaths-4279d2b03175.

14. Kadivar A. Corona's footsteps in civil registration statistics. 2020 May 19. [cited 2020 Jun 15]. Available from: https://blog.statsminute.ir/civilreg-deathstats-98/. Persian.

15. Olson DR, Huynh M, Fine A, Baumgartner J, Castro A, Chan HT, et al. Preliminary estimate of excess mortality during the COVID-19 outbreak-New York City, March 11-May 2, 2020. 2020. doi:10.15585/mmwr.mm6919e5.

16. Simonsen L, Clarke MJ, Williamson GD, Stroup DF, Arden NH, Schonberger LB. The impact of influenza epidemics on mortality: introducing a severity index. American journal of public health. 1997;87(12):1944-1950. doi:10.2105/ajph.87.12.1944.

17. National Organization for Civil Registration (NOCR). Registered Deaths by Province by Sex. Tehran, Iran: 2020 January 20. [updated 2020 May; cited 2020 May 8]. Available from: https://www.sabteahval.ir/avej/Page. aspx?mId=49826\&ID=2182\&Pag $\mathrm{e}=$ Magazines/SquareshowMagazine. Persian.

18. Welsch RE, Kuh E. Technical Report 923-77: Linear Regression Diagnostics. Cambridge, MA: Sloan School of Management, Massachusetts Institute of Technology. 1977:775.

19. Serfling RE. Methods for current statistical analysis of excess pneumonia-influenza deaths. Public health reports. 1963;78(6):494. doi: $10.2307 / 4591848$.

20. Muscatello DJ, Cretikos MA, MacIntyre CR. All-cause mortality during first wave of pandemic (H1N1) 2009, New South Wales, Australia, 2009. Emerging infectious diseases. 2010;16(9):1396. doi:10.3201/eid1609.091723

21. StataCorp. Stata Statistical Software. Release 15. College Station, TX: StataCorp LLC; 2017.

22. Ministry of Health and Medical Education (MoHME). Coronavirus News. [cited 2020 March 20]. Available from: https://behdasht.gov.ir/اخبار-كرونا-ويروس. Persian.

23. Iranian Legal Medicine Organization (ILMO). Motor Vehicle Crash Statistics. Tehran, Iran. [updated 2020 May; cited 2020 May 13] Available from: https://www.lmo.ir/web directory/53999تصادفات.html. Persian.

24. United Nations Statistical Division (USDN). Demographic and Social Statistics. [updated 2017 December; cited 2020 May 28] Available from: https://unstats.un.org/unsd/demographic-social/crvs/ index.cshtml.

25. World Health Organization (WHO). Influenza update - 354. 2019.

26. World Health Organization (WHO). Influenza update - 355. 2019.

27. World Health Organization (WHO). Influenza update - 356. 2019.

28. World Health Organization (WHO). Influenza update - 357. 2019.

29. World Health Organization (WHO). Influenza update - 358. 2020.

30. Nearly 200 to 225 people killed in November protests, minister suggests. Tehran Times; 2020 May 31. [cited 2020 June 2]. Available from: https://www.tehrantimes.com/news/448411/Nearly-200-to-225people-killed-in-November-protests-minister.

31. 230 people killed in November protests: MP. Tehran Times; 2020 June 2. [cited 2020 June 2]. Available from: https://www.tehrantimes.com/news/448457/230-people-killed-in-Nov ember-protests-MP

32. Amnesty International. Iran: Details of 304 deaths in crackdown on November 2019 protests. 2020 May 20. [cited 2020 May 30]. Available from: https://www.amnesty.org/en/documents/mde13/ 2308/2020/en/.

33. Reuters staff. Special Report: Iran's leader ordered crackdown on unrest - 'Do whatever it takes to end it'. Reuters; 2019 December 23 [cited 2020 May 30]. Available from: https://www.reuters.com/article/ us-iran-protests-specialreport/special-report-irans-leader-ordered-crack down-on-unrest-do-whatever-it-takes-to-end-it-idUSKBN1YR0QR.

34. huaxia. Full text of Wuhan's notification on revising numbers of confirmed COVID-19 cases, deaths. Xinhua; 2020 April 17. [cited 2020 June 2]. Available from: http://www.xinhuanet.com/english/ 2020-04/17/c_138984653.htm. 